\title{
Recognizing malicious software behaviors with tree automata inference
}

\author{
Domagoj Babić · Daniel Reynaud • Dawn Song
}

(C) Springer Science+Business Media, LLC 2012

\begin{abstract}
We explore how formal methods and tools of the verification trade could be used for malware detection and analysis. In particular, we propose a new approach to learning and generalizing from observed malware behaviors based on tree automata inference. Our approach infers $k$-testable tree automata from system call dataflow dependency graphs. We show how inferred automata can be used for malware recognition and classification.
\end{abstract}

Keywords Tree automata inference $\cdot$ Behavioral malware detection

\section{Introduction}

Over the last several decades, the IT industry advanced almost every aspect of our lives (including health care, banking, traveling) and industrial manufacturing. The tools and techniques developed in the computer-aided verification community played an important role in that advance, changing the way we design systems and improving the reliability of industrial hardware, software, and protocols.

This paper is an extended journal version of [3]. The extensions include additional experimental results and a more thorough discussion.

This material is based upon work partially supported by the National Science Foundation under Grants No. 0832943, 0842694, 0842695, 0831501, 0424422, by the Air Force Research Laboratory under Grant No. P010071555, by the Office of Naval Research under MURI Grant No. N000140911081, and by the MURI program under AFOSR Grants No. FA9550-08-1-0352 and FA9550-09-1-0539. The work of the first author is also supported by the Natural Sciences and Engineering Research Council of Canada PDF fellowship.

D. Babić $(\varangle) \cdot$ D. Reynaud · D. Song

Computer Science Division, University of California, Berkeley, CA 94720-1776, USA

e-mail: babic@cs.berkeley.edu

D. Reynaud

e-mail: reynaud@cs.berkeley.edu

D. Song

e-mail: dawnsong@cs.berkeley.edu

Published online: 11 April 2012 
In parallel, another community made a lot of progress exploiting software flaws for various nefarious purposes, especially for illegal financial gain. Their inventions are often ingenious botnets, worms, and viruses, commonly known as malware. Malware source code is rarely available and malware is regularly designed so as to thwart static analysis through the use of obfuscation, packing, and encryption [36].

For the above mentioned reasons, detection, analysis, and classification of malware are difficult to formalize, explaining why the verification community has mostly avoided, with some notable exceptions (e.g., $[8,19])$, the problem. However, the area is in a dire need of new approaches based on strong formal underpinnings, as less principled techniques, like signature-based detection, are becoming insufficient. Recently, we have been experiencing a flood of malware [33], while the recent example of Stuxnet (e.g., [29]) shows that industrial systems are as vulnerable as our every-day computers.

In this paper, we show how formal methods, more precisely tree automata inference, can be used for capturing the essence of malicious behaviors, and how such automata can be used to detect behaviors similar to those observed during the training phase. First, we execute malware in a controlled environment to extract dataflow dependencies among executed system calls (syscalls) using dynamic taint analysis [6,31]. The main way for programs to interact with their environment is through syscalls, which are broadly used in the security community as a high-level abstraction of software behavior [14, 25, 34]. The dataflow dependencies among syscalls can be represented by an acyclic graph, in which nodes represent executed syscalls, and there is an edge between two nodes, say $s_{1}$ and $s_{2}$, when the result computed by $s_{1}$ (or a value derived from it) is used as a parameter of $s_{2}$. Second, we use tree automata inference to learn an automaton recognizing a set of graphs. The entire process is completely automated.

The inferred automaton captures the essence of different malicious behaviors. We show that we can adjust the level of generalization with a single tunable factor and how the inferred automaton can be used to detect likely malicious behaviors, as well as for malware classification. We summarize the contributions of our paper as follows:

- Expansion of dependency graphs into trees causes exponential blowup in the size of the graph, similarly as with eager inlining of functions during static analysis. We found that a class of tree languages, namely $k$-testable tree languages [37] can be inferred directly from dependency graphs, avoiding the expansion to trees.

- We improve upon the prior work on inference of $k$-testable tree languages by providing an $\mathscr{O}(k N)$ algorithm, where $k$ is the size of the pattern and $N$ is the size of the graph used for inference.

- We show how inferred automata can be used for detecting likely malicious behaviors and for malware classification. To our knowledge, this is the first work applying the theory of tree automata inference to malware analysis. We provide experimental evidence that our approach is both feasible and useful in practice.

\section{Related work}

\subsection{Tree automata inference}

Inference of minimal finite state automata from both positive and negative examples is known to be NP-complete [18]. Thus, inferring a single minimal classifier for millions of new malware samples that appear each year might be infeasible. Inferring a non-minimal 
classifier is feasible, but the classifier could be too large to be useful in practice. In this paper, we focus on a less expressive family of languages, for which minimal automata can be efficiently identified from positive examples only. ${ }^{1}$

A subclass of regular tree languages - $k$-testable tree languages [37]—is identifiable in the limit from positive examples only. These languages are defined in terms of a finite set of $k$-level-deep tree patterns. The $k$ factor effectively determines the level of abstraction, which can be used as a knob to regulate the ratio of false positives (goodware detected as malware) and false negatives (undetected malware). The patterns partition dependency graphs into a finite number of equivalence classes, inducing a state-minimal automaton. The automata inferred from positive (malware) examples could be further refined using negative (goodware) examples. Such a refinement is conceptually simple, and does not increase the inference complexity, because of the properties of $k$-testable tree languages. We leave such a refinement for future work.

A number of papers focused on $k$-testable tree automata inference. Garcia and Vidal [16] proposed an $\mathscr{O}(k P N)$ inference algorithm, where $k$ is the size of the pattern, $P$ the total number of possible patterns, and $N$ the size of the input used for inference. Many patterns might not be present among the training samples, so rather than enumerating all patterns, [15] and [24] propose very similar algorithms that use only the patterns present in the training set. Their algorithms are somewhat complex to implement as they require computation of three different sets (called roots, forks, and leaves). Their algorithms are $\mathscr{O}\left(M^{k} N \log (N)\right)$, where $M$ is the maximal arity of any alphabet symbol in the tree language. We derive a simpler algorithm, so that computing forks and leaves becomes unnecessary. The complexity of our algorithm is $\mathscr{O}(k N)$, thanks to an indexing trick that after performing $k$ iterations over the training sample builds an index for finding patterns in the training set. Patterns in the test set can be located in the index table in amortized time linear in the size of the pattern. In our application-malware analysis- the $k$ factor tends to be small $(\leq 5)$, so our algorithm can be considered linear-time.

\subsection{Malware analysis}

From the security perspective, several types of malware analysis are interesting: malware detection (i.e., distinguishing malware from goodware), classification (i.e., determining the family of malware to which a particular sample belongs), and phylogeny (i.e., forensic analysis of evolution of malware and common/distinctive features among samples). All three types of analyses are needed in practice: detection for preventing further infections and damage to the infected computers, and the other two analyses are crucial in development of new forms of protection, forensics, and attribution. In this paper, we focus on detection and classification.

The origins of the idea to use syscalls to analyze software can be traced to Forrest et al. [13], who used fixed-length sequences of syscalls for intrusion detection. Wagner and Dean [34] built non-deterministic push-down automata (NDPDA) accepting valid sequences of syscalls, obtained through static analysis of the source code. Such automata are then used for monitoring the execution of programs at runtime. If a runtime sequence of syscalls is rejected, that might signal an intrusion. The non-determinism of NDPDA posed significant challenges in the monitoring phase, but the idea of abstracting applications' behavior with syscalls was embraced by other researchers. For example, Christodorescu et al. [9]

\footnotetext{
${ }^{1}$ Positive examples are examples belonging to the language to be inferred, while negative examples are those not in the language.
} 
note that malware authors could easily reorder data-flow-independent syscalls, circumventing sequence-detection schemes, but if we analyze data-flow dependencies among syscalls and use such dependency graphs for detection, circumvention becomes harder. Data-flowdependent syscalls cannot be (easily) reordered without changing the semantics of the program. They compute a difference between sets of malware and goodware dependency graphs, and show how resulting graphs can be used to detect malicious behaviors. Such graph matching can detect only the exact behavioral patterns already seen in some training sample, but does not automatically generalize from observed behaviors, i.e., does not attempt to overapproximate the training set in order to detect similar, but not exactly the same behaviors. The approach is, however, fairly efficient, because the difference of two sets of ordered, directed, and acyclic dependency graphs can be computed in polynomial time. While the results we present in this paper do not take negative samples (obtained from goodware) into account, doing so would likely improve both the detection and reduce the false positive rate. It would be sufficient to exclude from the training set the graphs that appear in both goodware and malware training samples.

Fredrikson et al. [14] propose an approach that focuses on distinguishing features, rather than similarities among dependency graphs. First, they compute dependency graphs at runtime, declaring two syscalls, say $s_{1}$ and $s_{2}$, dependent, if the type and value of the value returned by $s_{1}$ are equal to the type and value of some parameter of $s_{2}$ and $s_{2}$ was executed after $s_{1}$. They extract significant behaviors from such graphs using structural leap mining, and then choose behaviors that can be combined together using concept analysis. In spite of a very coarse unsound approximation of the dependency graph and lack of automatic generalization, they report $86 \%$ detection rate on around 500 malware samples used in their experiments. We see their approach as complementary to ours: the tree-automata we infer from real dependency graphs obtained through taint analysis could be combined with leap mining and concept analysis, to improve their classification power.

Bonfante et al. [4] propose to unroll control-flow graphs obtained through dynamic analysis of binaries into trees. The obtained trees are more fine-grained than the syscall dependency graphs. The finer level of granularity could, in practice, be less susceptible to mimicry attacks (e.g., [35]), but is also easier to defeat through control-flow graph manipulations. The computed trees are then declared to be tree automata and the recognizer is built by a union of such trees. Unlike inference, the union does not generalize from the training samples and will recognize only behaviors that are the exact match for some previously observed behavior. The reported experiments include a large set of malware samples (over 10,000), but the entire set was used for training, and authors report only false positives on a set of goodware (2653 samples). Thus, it is difficult to estimate how well their approach would work for malware detection and classification.

\subsection{Taint analysis}

Dynamic taint analysis (DTA) [31] is a technique used to follow data flows in programs or whole systems at runtime. DTA can be seen as a single-path symbolic execution [23] over a very simple domain (set of taints). Its premises are simple: taint is a variable annotation introduced through taint sources, it is propagated through program execution according to some propagation rules until it reaches a taint sink. In our case, for instance, taint sources are the syscalls' output parameters, and taint sinks are the input parameters.

As will be discussed in detail later, our implementation is based on the binary rewriting framework Pin [27] and uses the taint propagation rules from Newsome and Song [31]. Since DTA must operate at the instruction-level granularity, it poses a significant runtime 
overhead. Our DTA implementation executes applications several thousand times slower than the native execution. Our position is that the speed of the taint analysis is less important than the speed of inference and recognition. The taint analysis can be run independently for each sample in parallel, the dependency graph extraction is linear with the length of each execution trace, and hardware-based information flow tracking has been proposed (e.g., [12, 32]) as a potential solution for improving performance. In contrast, inference techniques have to process all the samples in order to construct a single (or a small number of) recognizer(s). An average anti-virus vendor receives millions of new samples annually and the number of captured samples has been steadily growing over the recent years. Thus, we believe that scalability of inference is a more critical issue than the performance of the taint analysis.

In general, DTA both under- and over-approximates dependencies [6, 20]. The problem of under-tainting (i.e. an output appears untainted, but is actually derived from a tainted input) is generally caused by implicit flows due to control dependencies or interactions with the system. The inverse problem, over-tainting, generally comes from imprecision of the taint propagation rules, or difficulties that arise from working with machine code.

\section{Notation and terminology}

In this section, we introduce the notation and terminology used throughout the paper. First, we build up the basic formal machinery that allows us to define tree automata. Second, we introduce some notions that will help us define $k$-roots that can be intuitively seen as the top $k$ levels of a tree. Later, we will show how $k$-roots induce an equivalence relation used in our inference algorithm. Towards the end of this section, we introduce $k$-testable languages, less expressive than regular tree languages, but suitable for designing fast inference algorithms.

Let $\mathbb{N}$ be the set of natural numbers and $\mathbb{N}^{*}$ the free monoid generated by $\mathbb{N}$ with concatenation $(\cdot)$ as the operation and the empty string $\epsilon$ as the identity. The prefix order $\leq$ is defined as: $u \leq v$ for $u, v \in \mathbb{N}^{*}$ iff there exists $w \in \mathbb{N}^{*}$ such that $v=u \cdot w$. For $u \in \mathbb{N}^{*}, n \in \mathbb{N}$, the length $|u|$ is defined inductively: $|\epsilon|=0,|u \cdot n|=|u|+1$. We say that a set $S$ is prefixclosed if $u \leq v \wedge v \in S \Rightarrow u \in S$. A tree domain is a finite non-empty prefix-closed set $D \subset \mathbb{N}^{*}$ satisfying the following property: if $u \cdot n \in D$ then $\forall 1 \leq j \leq n . u \cdot j \in D$.

A ranked alphabet is a finite set $\mathscr{F}$ associated with a finite ranking relation arity $\subseteq$ $\mathscr{F} \times \mathbb{N}$. Define $\mathscr{F}_{n}$ as a set $\{f \in \mathscr{F} \mid(f, n) \in$ arity $\}$. The set $T(\mathscr{F})$ of terms over the ranked alphabet $\mathscr{F}$ is the smallest set defined by:

1. $\mathscr{F}_{0} \subseteq T(\mathscr{F})$

2. if $n \geq 1, f \in \mathscr{F}_{n}, t_{1}, \ldots, t_{n} \in T(\mathscr{F})$ then $f\left(t_{1}, \ldots, t_{n}\right) \in T(\mathscr{F})$

Each term can be represented as a finite ordered tree $t: D \rightarrow \mathscr{F}$, which is a mapping from a tree domain into the ranked alphabet such that $\forall u \in D$ :

1. if $t(u) \in \mathscr{F}_{n}, n \geq 1$ then $\{j \mid u \cdot j \in D\}=\{1, \ldots, n\}$

2. if $t(u) \in \mathscr{F}_{0}$ then $\{j \mid u \cdot j \in D\}=\varnothing$

As usual in the tree automata literature (e.g., [10]), we use the letter $t$ (possibly with various indices) both to represent a tree as a mathematical object and to name a relation that maps an element of a tree domain to the corresponding alphabet symbol. An example of a tree with its tree domain is given in Fig. 1.

The set of all positions in a particular tree $t$, i.e., its domain, will be denoted $\operatorname{dom}(t)$. A subtree of $t$ rooted at position $u$, denoted $t / u$ is defined as $(t / u)(v)=t(u \cdot v)$ and 
Fig. 1 An example of a tree $t$ and its tree domain. $\operatorname{dom}(t)=\{1,11,111,112,12,13,131\}$, $\mathscr{F}=\{f, g, h, a, b\},\|t\|=3$, $t(1)=f, t / 131=b$

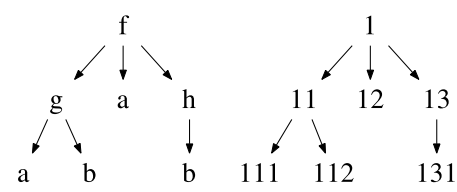

$\operatorname{dom}(t / u)=\{v \mid u \cdot v \in \operatorname{dom}(t)\}$. We generalize the $\operatorname{dom}$ operator to sets as: $\operatorname{dom}(S)=$ $\{\operatorname{dom}(u) \mid u \in S\}$. The height of a tree $t$, denoted $\|t\|$, is defined as:

$$
\|t\|=\max (\{|u| \text { such that } u \in \operatorname{dom}(t)\})
$$

Let $\Xi=\left\{\xi_{f} \mid f \in \bigcup_{i>0} \mathscr{F}_{i}\right\}$ be a set of new nullary symbols such that $\Xi \cap \mathscr{F}=\emptyset$. The $\Xi$ set will be used as a set of placeholders, such that $\xi_{f}$ can be substituted only with a tree $t$ whose position one (i.e., the head) is labeled with $f$, i.e., $t(1)=f$. Let $T(\Xi \cup \mathscr{F})$ denote the set of trees over the ranked alphabet and placeholders. For $t, t^{\prime} \in T(\Xi \cup \mathscr{F})$, we define the link operation $t \sharp t^{\prime}$ by:

$$
\left(t \sharp t^{\prime}\right)(n)= \begin{cases}t(n) & \text { if } n \in \operatorname{dom}(t) \wedge\left(t(n) \notin \Xi \vee\left(t(n)=\xi_{f} \wedge t^{\prime}(1) \neq f\right)\right) \\ t^{\prime}(z) & \text { if } n=y \cdot z, t(y)=\xi_{t^{\prime}(1)}, y \in \operatorname{dom}(t), z \in \operatorname{dom}\left(t^{\prime}\right)\end{cases}
$$

For any two trees, $t, t^{\prime} \in T(\mathscr{F})$, the tree quotient $t^{-1} t^{\prime}$ is defined by:

$$
t^{-1} t^{\prime}=\left\{t^{\prime \prime} \in T(\Xi \cup \mathscr{F}) \mid t^{\prime}=t^{\prime \prime} \sharp t\right\}
$$

The tree quotient operation can be extended to sets, as usual: $t^{-1} S=\left\{t^{-1} t^{\prime} \mid t^{\prime} \in S\right\}$. For any $k \geq 0$, define $k$-root of a tree $t$ as:

$$
\operatorname{root}_{k}(t)= \begin{cases}t & \text { if } t(1) \in \mathscr{F}_{0} \\ \xi_{f} & \text { if } f=t(1), f \in \bigcup_{i>0} \mathscr{F}_{i}, k=0 \\ f\left(\operatorname{root}_{k-1}\left(t_{1}\right), \ldots, \operatorname{root}_{k-1}\left(t_{n}\right)\right) & \text { if } t=f\left(t_{1}, \ldots, t_{n}\right),\|t\|>k>0\end{cases}
$$

A finite deterministic bottom-up tree automaton (FDTA) is defined as a tuple $(Q, \mathscr{F}, \delta, F)$, where $Q$ is a finite set of states, $\mathscr{F}$ is a ranked alphabet, $F \subseteq Q$ is the set of final states, and $\delta=\bigcup_{i} \delta_{i}$ is a set of transition relations defined as follows: $\delta_{0}: \mathscr{F}_{0} \rightarrow Q$ and for $n>0$, $\delta_{n}:\left(\mathscr{F}_{n} \times Q^{n}\right) \rightarrow Q$.

The $k$-testable in the strict sense ( $k$-TSS) languages [24] are intuitively defined by a set of tree patterns allowed to appear as the elements of the language. The following theorem is due to López et al. [26, Theorem 6.1]:

Theorem 1 Let $\mathscr{L} \subseteq T(\mathscr{F})$. $\mathscr{L}$ is a $k$-TSS iff for any trees $t_{1}, t_{2} \in T(\mathscr{F})$ such that $\operatorname{root}_{k}\left(t_{1}\right)=\operatorname{root}_{k}\left(t_{2}\right)$, when $t_{1}^{-1} \mathscr{L} \neq \emptyset \wedge t_{2}^{-1} \mathscr{L} \neq \emptyset$, then it follows that $t_{1}^{-1} \mathscr{L}=t_{2}^{-1} \mathscr{L}$.

We choose López et al.'s theorem as a definition of $k$-TSS languages. Other definitions in the literature $[15,24]$ define $k$-TSS languages in terms of three sets; leaves, roots, and forks. Forks are roots that have at least one placeholder as a leaf. Theorem 1 shows that such more complex definitions are unnecessary. Intuitively, the theorem says that within the language, any two subtrees that agree on the top $k$ levels are interchangeable, meaning that a bottomup tree automaton has to remember only a finite amount of history. In the next section, we show that we can define an equivalence relation inducing an automaton accepting a $k$-TSS language using only our definition of the $k$-root, as expected from Theorem 1 . 


\section{$4 k$-Testable tree automata inference}

\subsection{Congruence relation}

We begin with our definition of the equivalence relation that is used to induce a stateminimal automaton from a set of trees. The equivalence relation, intuitively, compares trees up to $k$ levels deep, i.e., compares $k$-roots.

Definition 1 (Root Equivalence Relation $\sim_{k}$ ) For some $k \geq 0$, two trees $t_{1}, t_{2} \in T(\mathscr{F})$ are root-equivalent with degree $k$, denoted $t_{1} \sim_{k} t_{2}$, if $\operatorname{root}_{k}\left(t_{1}\right)=\operatorname{root}_{k}\left(t_{2}\right)$.

Lemma 1 The $\sim_{k}$ relation is a congruence (monotonic equivalence) relation of finite index.

Proof (Sketch) It is obvious that $\sim_{k}$ is an equivalence relation (reflexive, symmetric, and transitive), and here we show that it is also monotonic, and therefore a congruence. Suppose $t_{1}=f\left(t_{11}, \ldots, t_{1 n}\right)$ and $t_{2}=f\left(t_{21}, \ldots, t_{2 n}\right)$, such that $\operatorname{root}_{k}\left(t_{1} / i\right)=\operatorname{root}_{k}\left(t_{2} / i\right)$ for all $1 \leq i \leq n$. First, note that if $k>0$ and $\operatorname{root}_{k}(t)=\operatorname{root}_{k}\left(t^{\prime}\right)$, then $\operatorname{root}_{k-1}(t)=\operatorname{root}_{k-1}\left(t^{\prime}\right)$. According to the definition of root $_{k}$, for $k>0$ we obtain:

$$
\begin{aligned}
\operatorname{root}_{k}\left(t_{1}\right) & \\
\quad=f\left(\operatorname{root}_{k-1}\left(t_{11}\right), \ldots, \operatorname{root}_{k-1}\left(t_{1 n}\right)\right) & \text { By definition of } \operatorname{root}_{k} \\
=f\left(\operatorname{root}_{k-1}\left(t_{21}\right), \ldots, \operatorname{root}_{k-1}\left(t_{2 n}\right)\right) & \text { By inductive hypothesis } \\
=\operatorname{root}_{k}\left(t_{2}\right) & \text { By definition of } \operatorname{root}_{k}
\end{aligned}
$$

The $k=0$ case is trivial, as $\operatorname{root}_{0}\left(t_{1}\right)=\xi_{f}=\operatorname{root}_{0}\left(t_{2}\right)$.

The size of a $k$-root is bounded by $M^{k}$, where $M=\max \left(\left\{n \mid \mathscr{F}_{n} \in F, \mathscr{F}_{n} \neq \emptyset\right\}\right)$. Each position $u$ in the $k$-root's domain can be labeled with at most $\left|\mathscr{F}_{\operatorname{arity}(t(u))}\right|$ symbols. Thus, root $_{k}$ generates a finite number of equivalence classes, i.e., is of finite index.

As a consequence of Lemma 1, inference algorithms based on the root equivalence relation need not propagate congruences using union-find [11] algorithms, as the root equivalence relation is a congruence itself.

Definition $2\left(\sim_{k}\right.$-induced Automaton) Let $T^{\prime} \subseteq T(\mathscr{F})$ be a finite set of finite trees. The $A^{\sim_{k}}\left(T^{\prime}\right)=(Q, \mathscr{F}, \delta, F)$ automaton induced by the root equivalence relation $\sim_{k}$ is defined as:

$$
\begin{aligned}
Q & =\left\{\operatorname{root}_{k}\left(t^{\prime}\right) \mid \exists t \in T^{\prime} . \exists u \in \operatorname{dom}\left(T^{\prime}\right) . t^{\prime}=t / u\right\} \\
F & =\left\{\operatorname{root}_{k}(t) \mid t \in T^{\prime}\right\} \\
\delta_{0}(f) & =f \text { for } f \in \mathscr{F}_{0} \\
\delta_{n}\left(f, \operatorname{root}_{k}\left(t_{1}\right), \ldots, \operatorname{root}_{k}\left(t_{n}\right)\right) & =\operatorname{root}_{k}\left(f\left(t_{1}, \ldots, t_{n}\right)\right) \quad \text { for } n \geq 1, f \in \mathscr{F}_{n}
\end{aligned}
$$

Corollary 1 (Containment) From the definition it follows that $\forall k \geq 0 . T^{\prime} \subseteq \mathscr{L}\left(A^{\sim_{k}}\left(T^{\prime}\right)\right)$. In other words, the $\sim_{k}$-induced automaton abstracts the set of trees $T^{\prime}$.

Theorem $2 \mathscr{L}\left(A^{\sim k}\right)$ is a $k$-TSS language.

Proof We need to prove that $\forall t_{1}, t_{2} \in T(\mathscr{F}), k \geq 0 . \operatorname{root}_{k}\left(t_{1}\right)=\operatorname{root}_{k}\left(t_{2}\right) \wedge t_{1}^{-1} \mathscr{L}\left(A^{\sim_{k}}\right) \neq$ $\emptyset \wedge t_{2}^{-1} \mathscr{L}\left(A^{\sim_{k}}\right) \neq \emptyset \Rightarrow t_{1}^{-1} \mathscr{L}\left(A^{\sim_{k}}\right)=t_{2}^{-1} \mathscr{L}\left(A^{\sim_{k}}\right)$. Suppose the antecedent is true, but the consequent is false, i.e., $t_{1}^{-1} \mathscr{L}\left(A^{\sim_{k}}\right) \neq t_{2}^{-1} \mathscr{L}\left(A^{\sim_{k}}\right)$. Then there must exist $t$ such that $t \sharp t_{1} \in$ 
$\mathscr{L}\left(A^{\sim_{k}}\right)$ and $t \sharp t_{2} \notin \mathscr{L}\left(A^{\sim_{k}}\right)$. Let $u$ be the position of $\xi_{t_{2}(1)}$, i.e., $\left(t \sharp t_{2}\right) / u=t_{2}$. Without loss of generality, let $t$ be the tree with minimal $|u|$. Necessarily, $|u|>1$, as otherwise $t_{1}^{-1} \mathscr{L}\left(A^{\sim k}\right)=\emptyset$. Let $u=w \cdot i, i \in \mathbb{N}$. We prove that $t \sharp t_{2}$ must be in $\mathscr{L}\left(A^{\sim k}\right)$, contradicting the initial assumption, by induction on the length of $w$.

Base case $(|w|=1)$ : Let $(t(w))(1)=f, f \in \mathscr{F}_{n}$. There are two subcases: $n=1$ and $n>1$. For $n=1$, the contradiction immediately follows, as $\delta\left(f, \operatorname{root}_{k}\left(t_{1}\right)\right)=\delta\left(f, \operatorname{root}_{k}\left(t_{2}\right)\right)$. For the $n>1$ case, observe that for all positions $w \cdot j$ such that $1 \leq j \leq n$ and $j \neq i$, $\left(t \sharp t_{1}\right) / w \cdot j=\left(t \sharp t_{2}\right) / w \cdot j=t / w \cdot j$. From that observation and $\operatorname{root}_{k}\left(t_{1}\right)=\operatorname{root}_{k}\left(t_{j}\right)$, it follows that

$$
\begin{aligned}
& \delta\left(\left(t \sharp t_{1} / w\right)(1), \operatorname{root}_{k}\left(t \sharp t_{1} / w \cdot 1\right), \ldots, \operatorname{root}_{k}\left(t \sharp t_{1} / w \cdot n\right)\right) \\
& \quad=\delta\left(\left(t \sharp t_{2} / w\right)(1), \operatorname{root}_{k}\left(t \sharp t_{2} / w \cdot 1\right), \ldots, \operatorname{root}_{k}\left(t \sharp t_{2} / w \cdot n\right)\right)
\end{aligned}
$$

Induction step $(|w|>1)$ : Let $w=w^{\prime} \cdot m, m \in \mathbb{N}$. From the induction hypothesis, we know that for all $m$, $\operatorname{root}_{k}\left(t \sharp t_{1} / w\right)=\operatorname{root}_{k}\left(t \sharp t_{2} / w\right)$, thus it follows:

$$
\begin{aligned}
& \delta\left(\left(t \sharp t_{1} / w^{\prime}\right)(1), \operatorname{root}_{k}\left(t \sharp t_{1} / w^{\prime} \cdot 1\right), \ldots, \operatorname{root}_{k}\left(t \sharp t_{1} / w^{\prime} \cdot n\right)\right) \\
& \quad=\delta\left(\left(t \sharp t_{2} / w^{\prime}\right)(1), \operatorname{root}_{k}\left(t \sharp t_{2} / w^{\prime} \cdot 1\right), \ldots, \operatorname{root}_{k}\left(t \sharp t_{2} / w^{\prime} \cdot n\right)\right)
\end{aligned}
$$

\section{Proposition 1 (Minimality) $A^{\sim_{k}}$ is state-minimal.}

Proof Follows from Myhill-Nerode Theorem [21, p. 72] and Lemma 1.

Minimality is not absolutely crucial for malware analysis in a laboratory setting, but it is important in practice, where antivirus tools can't impose a significant system overhead and have to react promptly to infections.

\section{Proposition 2 (Garcia [15]) $\mathscr{L}\left(A^{\sim k+1}\right) \subseteq \mathscr{L}\left(A^{\sim k}\right)$}

An important consequence of Garcia's theorem is that the $k$ factor can be used as an abstraction knob-the smaller the $k$ factor, the more abstract the inferred automaton. This tunability is particularly important in malware detection. One can't hope to design a classifier capable of perfect malware and goodware distinction. Thus, tunability of the false positive (goodware detected as malware) and false negative (undetected malware) ratios is crucial. More abstract automata will result in more false positives and fewer false negatives.

\subsection{Inference algorithm}

In this section, we present our inference algorithm, but before proceeding with the algorithm, we discuss some practical aspects of inference from data-flow dependency graphs. As discussed in Sect. 2, we use taint analysis to compute data-flow dependencies among executed syscalls at runtime. The result of that computation is not a tree, but an acyclic directed graph, i.e., a partial order of syscalls ordered by the data-flow dependency relation, and expansion of such a graph into a tree could cause exponential blowup. Thus, it would be more convenient to have an inference algorithm that operates directly on graphs, without expanding them into trees. 
Fig. 2 Folding a tree into a maximally-shared graph

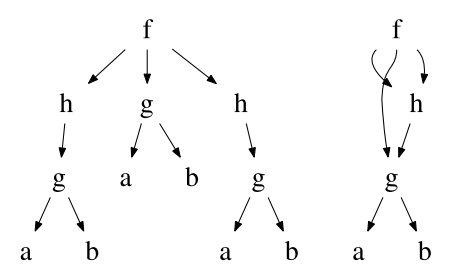

Fortunately, such an algorithm is only slightly more complicated than the one that operates on trees. In the first step, our implementation performs common subexpression elimination [1] on the dependency graph to eliminate syntactic redundancies. The result is a maximally-shared graph [2], i.e., an acyclic directed graph with shared common subgraphs. Figure 2 illustrates how a tree can be folded into a maximally-shared graph. In the second step, we compute a hash for each $k$-root in the training set. The hash is later used as a hash table key. Collisions are handled via chaining [11], as usual, but chaining is not described in the provided algorithms. The last step of the inference algorithm traverses the graph and folds it into a tree automaton, using the key computed in the second phase to identify equivalent $k$-roots, which are mapped to the same state.

To simplify the exposition, we shall use the formal machinery developed in Sect. 3 and present indexing and inference algorithms that work on trees. The extension to maximallyshared graphs is trivial and explained briefly later.

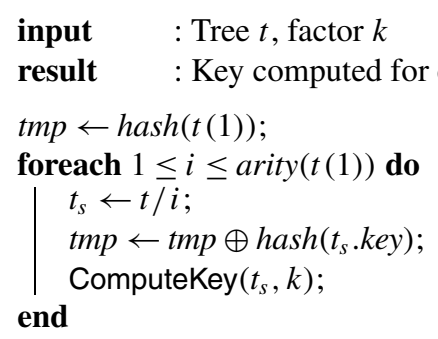

t.key $\leftarrow$ tmp

Algorithm 1 ComputeKey-Computing $k$-Root Keys (Hashes). The $\oplus$ operator can be any operator used to combine hashes, such as bitwise exclusive OR, with zero as the identity element. The hash $: \mathscr{F} \rightarrow \mathbb{N}$ function can be implemented as a string hash, returning an integral hash of the alphabet symbols

Algorithm 1 traverses tree $t$ in postorder (children before the parent). Every subtree has a field key associated with its head, and the field is assumed to be initially zero. If the algorithm is called once, for tree $t$, the key of the head of each subtree $t_{s}$ will consist only of the hash of the alphabet symbol labeling $t_{s}$, i.e., $\operatorname{hash}\left(t_{s}(1)\right)$. If the algorithm is called twice (on the same tree), the key of the head of each subtree will include the hash of its own label and the labels of its children, and so on. Thus, after $k$ calls to ComputeKey, the key of each node will be equal to its $k$-root key. Note that the temporary key, stored in the tmp variable, has to be combined with the children's $(k-1)$-root key. The algorithm can be easily extended to operate on maximally-shared graphs, but has to track visited nodes and visit each node only once in postorder. The complexity of the algorithm is $\mathscr{O}(k \cdot N)$, where $N$ is the size of 
the tree (or maximally-shared graph). For multi-rooted graphs (or when processing multiple trees), all roots can be connected by creating a synthetic super-root of all roots, and the algorithm is then called $k$ times with the super-root as the first operand.

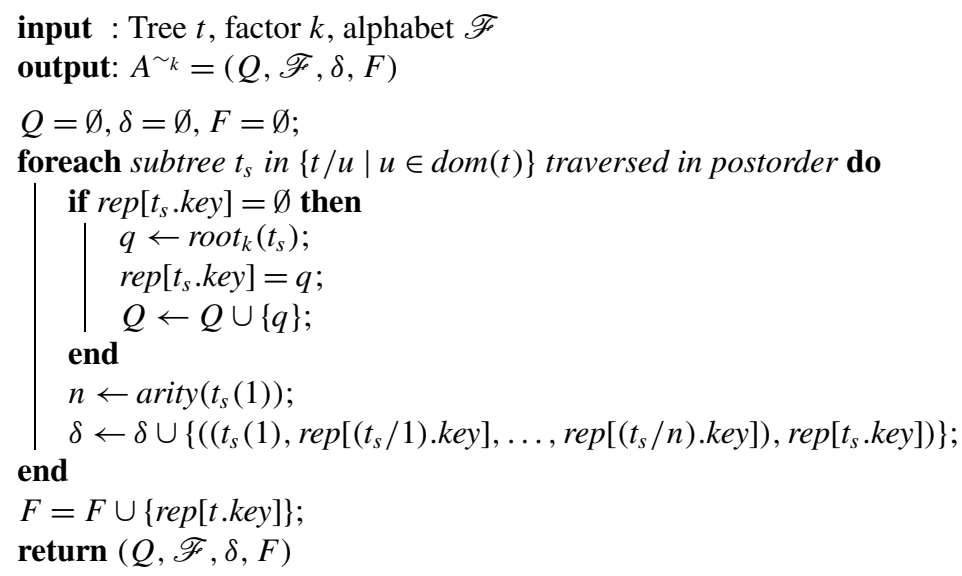

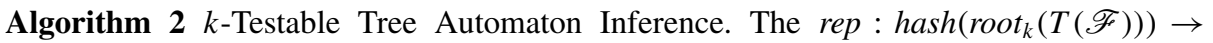
$\operatorname{root}_{k}(T(\mathscr{F}))$ hash map contains representatives of equivalence classes induced by $\sim_{k}$. Collisions are handled via chaining (not shown)

Algorithm 2 constructs the $A^{\sim_{k}}$ automaton. The tree (alternatively maximally-shared graph) used for training is traversed in postorder, and $k$-root of each subtree is used to retrieve the representative for each $\sim_{k}$-induced equivalence class. Multi-rooted graphs can be handled by introducing super-roots (as described before). Amortized complexity is $\mathscr{O}(k N)$, where $N$ is the size of the tree (or maximally-shared graph).

\section{Implementation}

\subsection{Taint analysis}

We use Pin [27] to perform instruction-level tracing and analysis. Pin is a dynamic binary instrumentation framework that allows program monitoring and rewriting only in user space, which prevents us from propagating taints through syscalls in the kernel space. One possible solution would be to declare all syscalls' input parameters to be taint sinks, and all output parameters to be taint sources. Unfortunately, the kernel interface for the Windows $\mathrm{XP}$ operating system is only partially documented. To work around this problem, we use the libwst library by Martignoni and Paleari [28] to automatically extract and parse parameters of Windows syscalls. With libwst, we find out the number, type, and directionality (in/out) of parameters. The reverse-engineered parameters are then used as an input-output specification of syscalls. After each return from a syscall, we walk the stack and mark any location pointed to by an out parameter as tainted with a new taint mark. At syscall entry (i.e., just before our tool loses control), we walk the stack and check if taint has reached any of its in parameters. Since each taint mark can be traced back to a unique out parameter, the set 
of dependencies for an in parameter corresponds exactly to the set of its taint marks. We approximate the leaves of the dependency graph (i.e., input parameters not returned by any syscall) with their types. A more precise approach, left for future work, would be to use the actual values.

Ideally, each malware sample would run unencumbered in the environment targeted by its authors. According to conventional wisdom, most malware samples target Windows XP, so we set it up with the latest service pack in a VirtualBox virtual machine with no network connection and only one user with administrative rights. Although the lack of network connection might prevent some samples from executing their payload, such a precaution is necessary to avoid spreading the infection. We infect the virtual machine via a shared folder. The physical machine used to run the dependency graph extraction experiments has a 2.66 GHz Intel Core i7 CPU and 8 GB RAM. After each run, we revert the virtual machine to a clean snapshot so that malware samples can not interfere with each other.

\subsection{Inference algorithm}

The inference algorithm is a relatively straightforward implementation of algorithms in Sect. 4.2, written in about 3200 lines of $\mathrm{C}++$ code. As explained before, after reading the dependency graphs, the implementation performs common subexpression elimination (CSE), computes $k$-root hashes (Algorithm 1), infers a $k$-testable tree automaton (Algorithm 2), and then runs the dependency graphs from the test set against that automaton. Both CSE and inference are done directly on dependency graphs, avoiding an expansion into trees.

\section{Experimental results}

\subsection{Benchmarks}

For the experiments, we use two sets of benchmarks: the malware and the goodware set. The malware set comprises 2631 samples pre-classified into 48 families. Each family contains 5-317 samples. We rely upon the classification of Christodorescu et al. [7] and Fredrikson et al. [14]. ${ }^{2}$ The classification was based on the reports from antivirus tools. For a small subset of samples, we confirmed the quality of classification using virustotal.com, a free malware classification service. However, without knowing the internals of those antivirus tools and their classification heuristics, we cannot evaluate the quality of the classification provided to us. Our classification experiments indicate that what the classification antivirus tools do might be somewhat ad-hoc. Table 1 shows the statistics for every family, while Table 2 shows goodware statistics. Table 3 gives some idea of how antivirus tools classify one randomly chosen sample.

The goodware set comprises 33 commonly used applications: AdobeReader, Apple SW Update, Autoruns, Battle for Wesnoth, Chrome, Chrome Setup, Firefox, Freecell, Freeciv, Freeciv server, GIMP, Google Earth, Internet Explorer, iTunes, Minesweeper, MSN Messenger, Netcat port listen and scan, NetHack, Notepad, OpenOffice Writer, Outlook Express, Ping, 7-zip archive, Skype, Solitaire, Sys info, Task manager, Tux Racer, uTorrent, VLC,

\footnotetext{
${ }^{2}$ The full set of malware contains 3136 samples, but we eliminated samples that were not executable, executable but not analyzable with Pin (i.e., MS-DOS, Win16, and POSIX subsystem executables), broken executables, and those that were incompatible with the version of Windows (XP) that we used for experiments.
} 
Table 1 Malware statistics per family. All dependency graphs were obtained by running each sample for $120 \mathrm{sec}$ in a controlled environment. The identifier that will be used in later graphs is given in the first column. The third column shows the number of samples per family. The Avg. column shows the average height of the dependency graphs across all the samples in the family. The Nodes column shows the total number of nodes in the dependency graph (after CSE). The Trees column shows the total number of different trees (i.e., roots of the dependency graph) across all the samples. The Max column gives the maximal height of any tree in the family

\begin{tabular}{|c|c|c|c|c|c|c|}
\hline ID & Family name & Samples & Avg. & Nodes & Trees & Max. \\
\hline 1 & ABU.Banload & 16 & 7.71 & 544 & 303 & 21 \\
\hline 2 & Agent & 42 & 8.86 & 965 & 593 & 27 \\
\hline 3 & Agent.Small & 15 & 8.88 & 950 & 588 & 27 \\
\hline 4 & Allaple.RAHack & 201 & 8.78 & 1225 & 761 & 44 \\
\hline 5 & Ardamax & 25 & 6.21 & 144 & 69 & 16 \\
\hline 6 & Bactera.VB & 28 & 7.09 & 333 & 177 & 28 \\
\hline 7 & Banbra.Banker & 52 & 13.97 & 1218 & 686 & 37 \\
\hline 8 & Bancos.Banker & 46 & 14.05 & 742 & 417 & 45 \\
\hline 9 & Banker & 317 & 17.70 & 2952 & 1705 & 43 \\
\hline 10 & Banker.Delf & 20 & 14.78 & 939 & 521 & 50 \\
\hline 11 & Banload.Banker & 138 & 19.38 & 2370 & 1332 & 152 \\
\hline 12 & BDH.Small & 5 & 5.82 & 348 & 199 & 21 \\
\hline 13 & BGM.Delf & 17 & 7.04 & 339 & 199 & 25 \\
\hline 14 & Bifrose.CEP & 35 & 11.17 & 1190 & 698 & 50 \\
\hline 15 & Bobax.Bobic & 15 & 8.98 & 859 & 526 & 30 \\
\hline 16 & DKI.PoisonIvy & 15 & 9.22 & 413 & 227 & 40 \\
\hline 17 & DNSChanger & 22 & 12.62 & 874 & 483 & 36 \\
\hline 18 & Downloader.Agent & 13 & 12.89 & 1104 & 613 & 49 \\
\hline 19 & Downloader.Delf & 22 & 10.76 & 1486 & 906 & 32 \\
\hline 20 & Downloader.VB & 17 & 10.80 & 516 & 266 & 29 \\
\hline 21 & Gaobot.Agobot & 20 & 17.54 & 1812 & 1052 & 45 \\
\hline 22 & Gobot.Gbot & 58 & 7.01 & 249 & 134 & 22 \\
\hline 23 & Horst.CMQ & 48 & 16.86 & 1030 & 541 & 42 \\
\hline 24 & Hupigon.ARR & 33 & 23.58 & 2388 & 1244 & 55 \\
\hline 25 & Hupigon.AWQ & 219 & 24.63 & 7225 & 3758 & 62 \\
\hline 26 & IRCBot.Sdbot & 66 & 16.51 & 3358 & 1852 & 47 \\
\hline 27 & LdPinch & 16 & 16.88 & 1765 & 1012 & 66 \\
\hline 28 & Lmir.LegMir & 23 & 9.00 & 1112 & 667 & 28 \\
\hline 29 & Mydoom & 15 & 5.78 & 484 & 305 & 20 \\
\hline 30 & Nilage.Lineage & 24 & 9.64 & 1288 & 657 & 83 \\
\hline 31 & Games.Delf & 11 & 8.44 & 971 & 632 & 22 \\
\hline 32 & Games.LegMir & 76 & 17.18 & 11892 & 8184 & 59 \\
\hline 33 & Games.Mmorpg & 19 & 7.00 & 654 & 478 & 25 \\
\hline 34 & OnLineGames & 23 & 7.30 & 718 & 687 & 16 \\
\hline 35 & Parite.Pate & 71 & 14.31 & 1420 & 816 & 36 \\
\hline 36 & Plemood.Pupil & 32 & 6.29 & 330 & 189 & 24 \\
\hline 37 & PolyCrypt.Swizzor & 43 & 10.32 & 415 & 213 & 30 \\
\hline 38 & Prorat.AVW & 40 & 23.47 & 1031 & 572 & 58 \\
\hline 39 & Rbot.Sdbot & 302 & 14.23 & 4484 & 2442 & 47 \\
\hline 40 & SdBot & 75 & 14.13 & 2361 & 1319 & 40 \\
\hline 41 & Small.Downloader & 29 & 11.93 & 2192 & 1216 & 34 \\
\hline 42 & Stration.Warezov & 19 & 9.76 & 1682 & 1058 & 34 \\
\hline 43 & Swizzor.Obfuscated & 27 & 21.75 & 1405 & 770 & 49 \\
\hline 44 & Viking.HLLP & 32 & 7.84 & 512 & 315 & 24 \\
\hline
\end{tabular}


Table 1 (Continued)

\begin{tabular}{llccrrr}
\hline ID & Family name & Samples & Avg. & Nodes & Trees & Max. \\
\hline 45 & Virut & 115 & 11.76 & 3149 & 1953 & 40 \\
46 & VS.INService & 17 & 11.42 & 307 & 178 & 37 \\
47 & Zhelatin.ASH & 53 & 12.14 & 1919 & 1146 & 39 \\
48 & Zlob.Puper & 64 & 15.16 & 2788 & 1647 & 90 \\
\hline
\end{tabular}

Win. Media Player, and WordPad. We deemed these applications to be representative of software commonly found on the average user's computer, from a number of different vendors and with a diverse set of behaviors. Also, we used two micro benchmarks: a HelloWorld program written in $\mathrm{C}$ and a file copy program. Micro-benchmarks produce few small dependency graphs and therefore might be potentially more susceptible to be misidentified for malware.

In behavioral malware detection, there is always a contention between the amount of time the behavior is observed and the precision of the analysis. For malware samples, which are regularly small pieces of software, we set the timeout to $120 \mathrm{sec}$ of running in our environment. We also tried the $800 \mathrm{~s}$ timeout on a small randomly selected subset of malware samples, without noticing a significant improvement in detection rates. For goodware, we wanted to study the impact of the runtime on the height and complexity of generated dependency graphs, and the impact of these differences on the false positive rates. Thus, we ran goodware samples for both 120 and $800 \mathrm{sec}$. To give some intuition of how that corresponds to the actual native runtime, it takes approximately $800 \mathrm{~s}$ in our DTA analysis environment for Acrobat Reader to open a document and display a window.

We noticed a general tendency that detection and classification tend to correlate positively with the average height of trees in samples used for training and testing. We provide the average heights in Tables 1 and 2, and heat maps providing a deeper insight into the distribution of the heights in Figs. 3, 4, and 5. The heat maps confirm our claim that malware is indeed simpler than goodware, at least when compared by their syscall dependency graphs. The majority of malware samples have dependency graphs with 30 or fewer levels and the deepest one has 70 levels, while many goodware samples ran for $120 \mathrm{~s}$ (Fig. 5) have graphs with more than 70 levels and three samples even had 100 levels. Running goodware for $800 \mathrm{~s}$ produces more deeper dependency graphs, as expected.

\subsection{Malware and goodware recognition}

For our malware recognition experiments, we chose at random $50 \%$ of the entire malware set for training, and used the rest and the entire goodware set as test sets. Training with $k=4$ took around $10 \mathrm{sec}$ for the entire set of 1315 training samples, and the time required for analyzing each test sample was less than the timing jitter (sub-second range). All the experiments were performed in Ubuntu 10.04, running in a VMware 7.1.3 workstation, running on Win XP Pro and dual-core $2.5 \mathrm{GHz}$ Intel machine with $4 \mathrm{~GB}$ of RAM. In Fig. 6, we show the results, using the goodware dependency graphs produced with an 800 sec timeout. The results obtained with a $120 \mathrm{sec}$ timeout are slightly worse and not shown. ${ }^{3}$

The detection works as follows. We run all the trees (i.e., roots of the dependency graph) in each test sample against the inferred automaton. First, we sort the trees by height, and

\footnotetext{
${ }^{3}$ The $120 \mathrm{sec}$ results are available in the full version available on the first author's web page.
} 
Table 2 Goodware statistics. For the description of other columns, see Table 1

\begin{tabular}{|c|c|c|c|c|c|c|c|c|c|}
\hline \multirow[b]{2}{*}{ ID } & \multirow[b]{2}{*}{ Application } & \multicolumn{4}{|c|}{$800 \mathrm{sec}$ Trace } & \multicolumn{4}{|c|}{$120 \mathrm{sec}$ Trace } \\
\hline & & Avg. & Nodes & Trees & Max. & Avg. & Nodes & Trees & Max. \\
\hline 1 & AdobeReader & 8.09 & 340 & 191 & 22 & 8.57 & 271 & 147 & 22 \\
\hline 2 & Apple SW Update & 13.74 & 561 & 317 & 51 & 20.87 & 293 & 151 & 51 \\
\hline 3 & Autoruns & 12.29 & 330 & 181 & 43 & 12.45 & 304 & 160 & 43 \\
\hline 4 & Battle for Wesnoth & 41.01 & 602 & 355 & 76 & 34.73 & 380 & 187 & 76 \\
\hline 5 & Chrome & 13.85 & 436 & 240 & 43 & 11.11 & 273 & 143 & 31 \\
\hline 6 & Chrome Setup & 5.19 & 148 & 74 & 17 & 5.19 & 148 & 74 & 17 \\
\hline 7 & Copy & 77.14 & 913 & 426 & 244 & 64.99 & 880 & 412 & 215 \\
\hline 8 & Firefox & 30.43 & 785 & 464 & 94 & 44.02 & 356 & 175 & 89 \\
\hline 9 & Freecell & 11.65 & 308 & 167 & 33 & 11.49 & 316 & 172 & 33 \\
\hline 10 & Freeciv & 28.48 & 472 & 241 & 75 & 37.14 & 300 & 137 & 72 \\
\hline 11 & Freeciv server & 11.46 & 300 & 177 & 30 & 11.62 & 297 & 174 & 30 \\
\hline 12 & GIMP & 30.97 & 681 & 359 & 86 & 36.33 & 299 & 134 & 69 \\
\hline 13 & Google Earth & 33.08 & 321 & 155 & 76 & 4.63 & 88 & 37 & 13 \\
\hline 14 & HelloWorld & 1.62 & 35 & 15 & 4 & 1.53 & 34 & 14 & 4 \\
\hline 15 & Internet Explorer & 10.58 & 572 & 319 & 49 & 13.08 & 279 & 139 & 45 \\
\hline 16 & iTunes & 48.81 & 852 & 457 & 120 & 32.05 & 404 & 217 & 75 \\
\hline 17 & Minesweeper & 10.85 & 304 & 167 & 30 & 10.72 & 305 & 167 & 30 \\
\hline 18 & MSN Messenger & 17.75 & 809 & 477 & 59 & 23.28 & 308 & 158 & 58 \\
\hline 19 & Netcat port listen & 65.08 & 997 & 494 & 241 & 67.05 & 873 & 413 & 225 \\
\hline 20 & Netcat port scan & 54.67 & 1123 & 597 & 241 & 65.69 & 882 & 420 & 225 \\
\hline 21 & NetHack & 4.94 & 124 & 63 & 15 & 4.94 & 124 & 63 & 15 \\
\hline 22 & Notepad & 9.68 & 350 & 198 & 30 & 10.69 & 298 & 165 & 30 \\
\hline 23 & OpenOffice Writer & 6.55 & 271 & 156 & 19 & 6.60 & 271 & 156 & 19 \\
\hline 24 & Outlook Express & 20.45 & 490 & 279 & 51 & 20.64 & 360 & 201 & 49 \\
\hline 25 & Ping & 11.82 & 535 & 317 & 34 & 12.19 & 360 & 197 & 34 \\
\hline 26 & 7-zip archive & 12.96 & 269 & 149 & 26 & 12.97 & 267 & 144 & 30 \\
\hline 27 & Skype & 1.38 & 31 & 12 & 3 & 1.38 & 31 & 12 & 3 \\
\hline 28 & Solitaire & 11.63 & 303 & 165 & 31 & 11.30 & 311 & 170 & 31 \\
\hline 29 & Sys. Info & 6.48 & 613 & 382 & 26 & 7.01 & 305 & 171 & 26 \\
\hline 30 & Task Manager & 11.28 & 513 & 307 & 35 & 11.94 & 343 & 196 & 35 \\
\hline 31 & TuxRacer & 14.11 & 441 & 261 & 44 & 15.53 & 279 & 157 & 39 \\
\hline 32 & uTorrent & 9.31 & 267 & 151 & 28 & 10.49 & 214 & 114 & 28 \\
\hline 33 & VLC & 12.92 & 325 & 178 & 38 & 12.76 & 295 & 159 & 38 \\
\hline 34 & Win. Media Player & 9.50 & 448 & 255 & 36 & 10.23 & 315 & 174 & 36 \\
\hline \multirow[t]{2}{*}{35} & WordPad & 8.33 & 420 & 235 & 28 & 8.52 & 262 & 147 & 27 \\
\hline & Average & 19.06 & 426 & 235 & 51 & 17.10 & 295 & 153 & 46 \\
\hline
\end{tabular}

then compute how many trees for each height are accepted by the automaton. Second, we score the sample according to the following function:

$$
\text { score }=\frac{\sum_{i} \frac{\text { accepted }_{i}}{\text { total }_{i}} * i}{\sum_{i} i}
$$


Table 3 Sample 3BC816C45FD461377E13A775AE8768A3 classification. Data obtained from Virustotal.com

\begin{tabular}{|c|c|c|c|}
\hline Antivirus & Classification & Antivirus & Classification \\
\hline AVG & Downloader.Generic4.GAF & AhnLab-V3 & Win-Trojan/Xema.variant \\
\hline AntiVir & TR/Agent.8192.123 & Antiy-AVL & Trojan/Win32.Agent.gen \\
\hline Avast & Win32\&Agent-GQA & Avast5 & Win32\&Agent-GQA \\
\hline BitDefender & Trojan.Downloader.Agent.AAH & $\begin{array}{l}\text { CAT- } \\
\text { QuickHeal }\end{array}$ & TrojanDownloader.Agent.aah \\
\hline ClamAV & Trojan.Downloader-6542 & Command & W32/Downldr2.COH \\
\hline Comodo & $\begin{array}{l}\text { TrojWare.Win } 32 . \\
\text { TrojanDownloader.Agent.AAH }\end{array}$ & nProtect & $\begin{array}{l}\text { Trojan-Downloader/ } \\
\text { W32.Agent.8192.K }\end{array}$ \\
\hline Emsisoft & Trojan-Dropper.Agent!IK & F-Prot & W32/Downldr2.COH \\
\hline F-Secure & Trojan.Downloader.Agent.AAH & GData & Trojan.Downloader.Agent.AAH \\
\hline Ikarus & Trojan-Dropper.Agent & Jiangmin & Trojan/PSW.GamePass.gir \\
\hline K7AntiVirus & Trojan-Downloader & Kaspersky & $\begin{array}{l}\text { Trojan-Downloader. } \\
\text { Win32.Agent.aah }\end{array}$ \\
\hline McAfee & Suspect-AB!3BC816C45FD4 & DrWeb & Adware.DealHelper \\
\hline Microsoft & $\begin{array}{l}\text { TrojanDownloader\& } \\
\text { Win32/Agent }\end{array}$ & NOD32 & $\begin{array}{l}\text { Win32/ } \\
\text { TrojanDownloader.Agent.AAH }\end{array}$ \\
\hline Norman & W32/Agent.ECGQ & PCTools & Trojan-Downloader.Agent.AAH \\
\hline Panda & Trj/Downloader.OEW & Prevx & Med. Risk Malware Downloader \\
\hline Rising & Trojan.Clicker.Win32.Small.nh & Sophos & Mal/Generic-L \\
\hline Symantec & Downloader & TheHacker & Trojan/Downloader.Agent.aah \\
\hline TrendMicro & TROJ_Generic & VirusBuster & Trojan.DL.Agent.TOR \\
\hline
\end{tabular}

where $i$ ranges from 1 to the maximal height of any tree in the test sample (the last column of Table 1), accepted $_{i}$ is the number of trees with height $i$ accepted by the automaton, and total $_{i}$ is the total number of trees with height $i$. The test samples that produce no syscall dependency graphs are assumed to have score zero.

The score can range from 0 to 1 . Higher score signifies a higher likelihood the sample is malicious. The ratio in the nominator of Eq. (1) is multiplied by the depth of the tree to filter out the noise from shallow trees, often generated by standard library functions, that have very low classification power.

The results turned out to be slightly better with an $800 \mathrm{sec}$ timeout than with the $120 \mathrm{sec}$ timeout, as the average height of dependency graphs was slightly larger. As expected, we found that with the rising $k$ factor (and therefore decreasing level of abstraction), the capability of inferred tree automaton to detect malware decreases, which obviously indicates the value of generalization achieved through tree automata inference. On the other hand, with the rising $k$ factor, the detection becomes more precise and therefore the false positive rate drops down. Thus, it is important to find the right level of abstraction. In our experiments, we determined that $k=4$ was the optimal abstraction level. The desired ratio between false positives and negatives can be adjusted by selecting the score threshold. All samples scoring above (resp. below) the threshold are declared malware (resp. goodware). For example, for $k=4$, timeout of $800 \mathrm{sec}$, and score 0.6, our approach reports two false positives (5\%) - Chrome setup and NetHack, and 270 false negatives $(20 \%)$, which corresponds to an $80 \%$ detection rate. For $k=4$, timeout of $800 \mathrm{sec}$, and score 0.6, our approach reports one additional false positive (System info), 


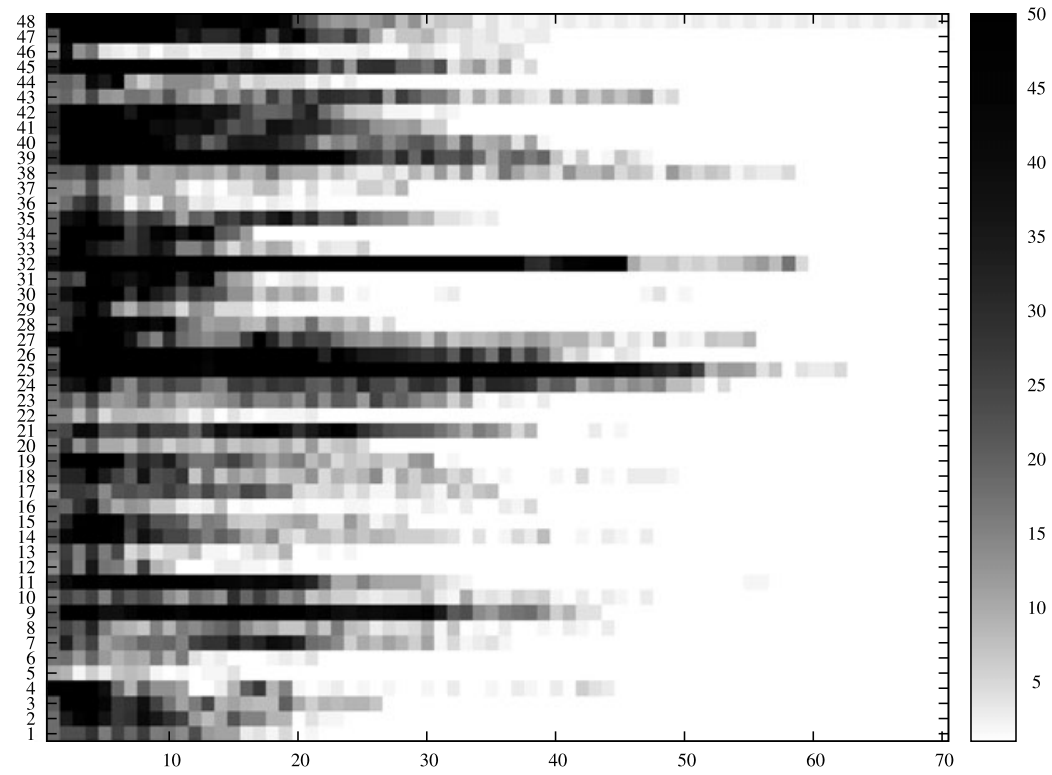

Fig. 3 Malware tree height heat map. The $x$ axis represents the tree height, while the $y$ axis lists malware families. The legend on the right is a color code for the number of trees observed with a particular height

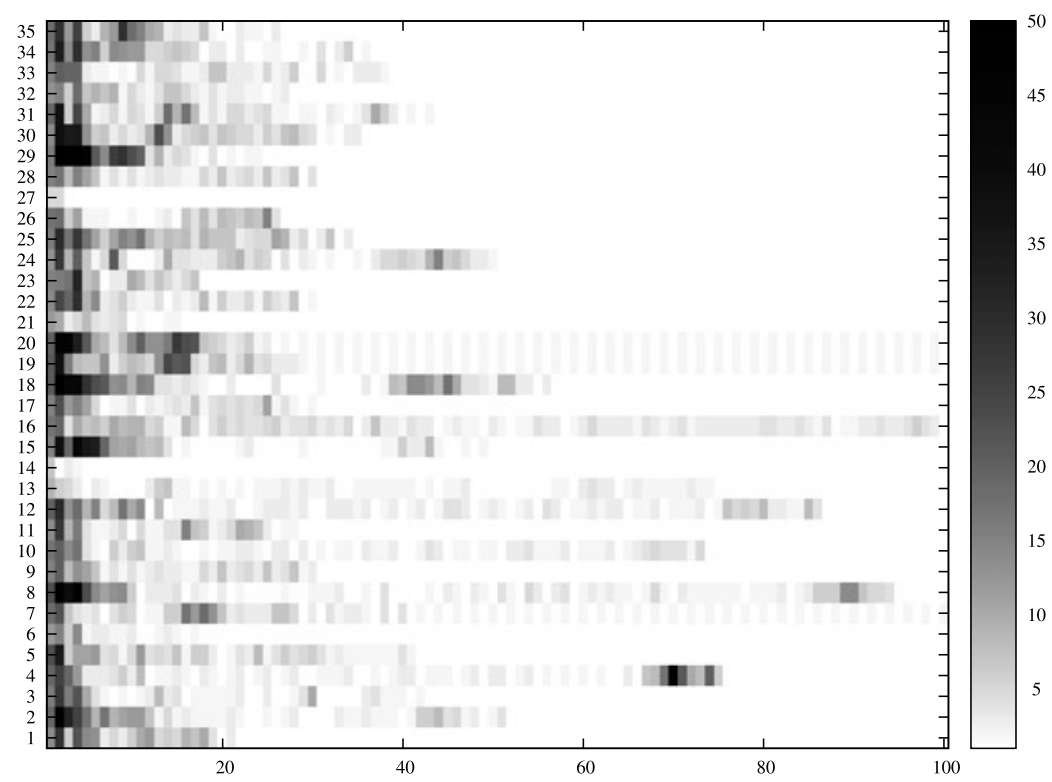

Fig. 4 Goodware (800 sec trace) Tree height heat map. The $x$ axis represents the goodware samples

and the same number of false negatives, although a few malware samples are somewhat closer to the threshold. Obviously, the longer the behavior is observed, the better the classification. 


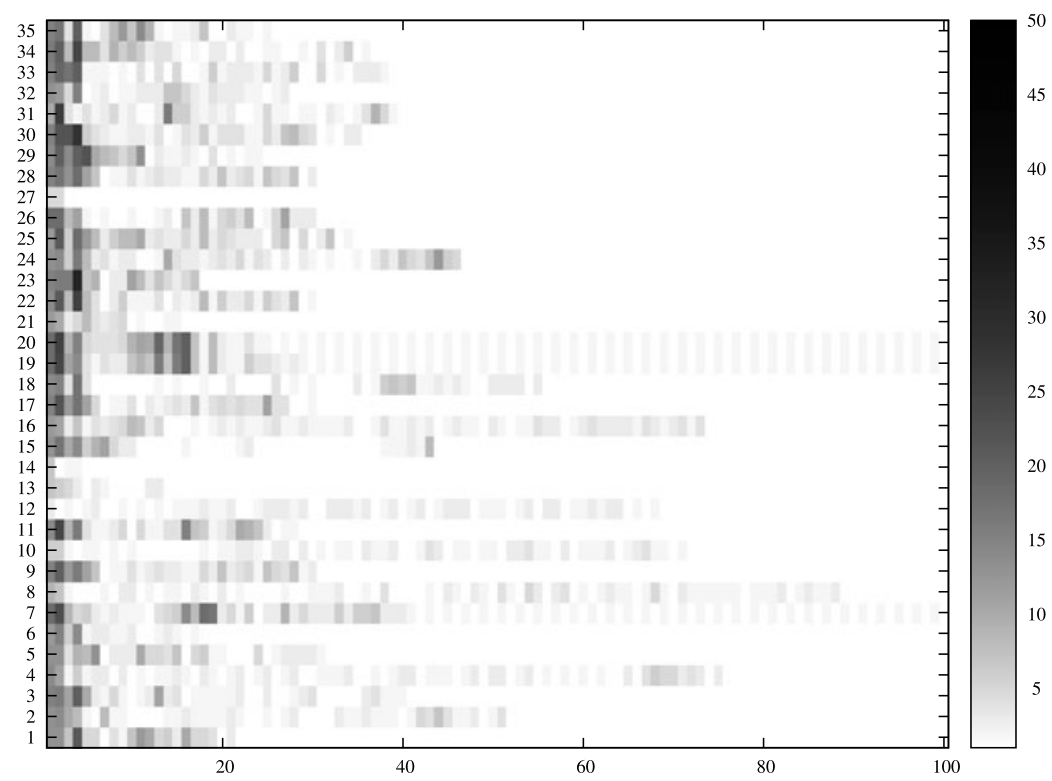

Fig. 5 Goodware (120 sec trace) Tree height heat map. The $x$ axis represents the goodware samples

It is interesting to notice that increasing the value of $k$ above 4 does not make a significant difference in (mis)detection rates. We ran the experiments with $k$ up to 10, but do not show the results as they are essentially the same as for $k=4$. From our preliminary analysis, it seems that generalization is effective when a sequence of dependent syscalls are executed within a loop. If two samples execute the same loop body a different number of times, our approach will be able to detect that. Changing $k$ effectively changes the window with which such loop bodies are detected. During the inference, it seems like one size (of $k$ ) does not fit all cases. We believe that by analyzing the repetitiveness of patterns in dependency graphs, we could detect the sizes of loop bodies much more accurately, and adjust the $k$ factor according to the size of the body, which should in turn improve the generalization capabilities of the inference algorithm. Many other improvements of our work are possible, as discussed later.

\subsection{Malware classification}

We were interested in investigating the classification power of inferred automata, so we did the following experiment. We divided at random each family into training and test sets of equal size. For each training set, we inferred a family-specific tree automaton. For each test set, we merge the dependency graphs for all the samples in the set, computing a single dependency graph, which is then analyzed with the inferred tree automaton. The scores are computed according to Eq. (1), with $k=3$. The only difference from the experiment done in the previous section is that the score is computed for the entire test set, not individual samples in the set. Results are shown in Fig. 7. The size of the dot in the graph is proportional to the computed score, the largest dots correspond to the score of 1.

Some classification results can be explained by looking at the previously given heat maps. For instance, family 25 has a large number of diverse graphs of different depths-almost the entire row 25 in Fig. 3 is black up to the depth of 50. Thus, it is not surprising that such a 


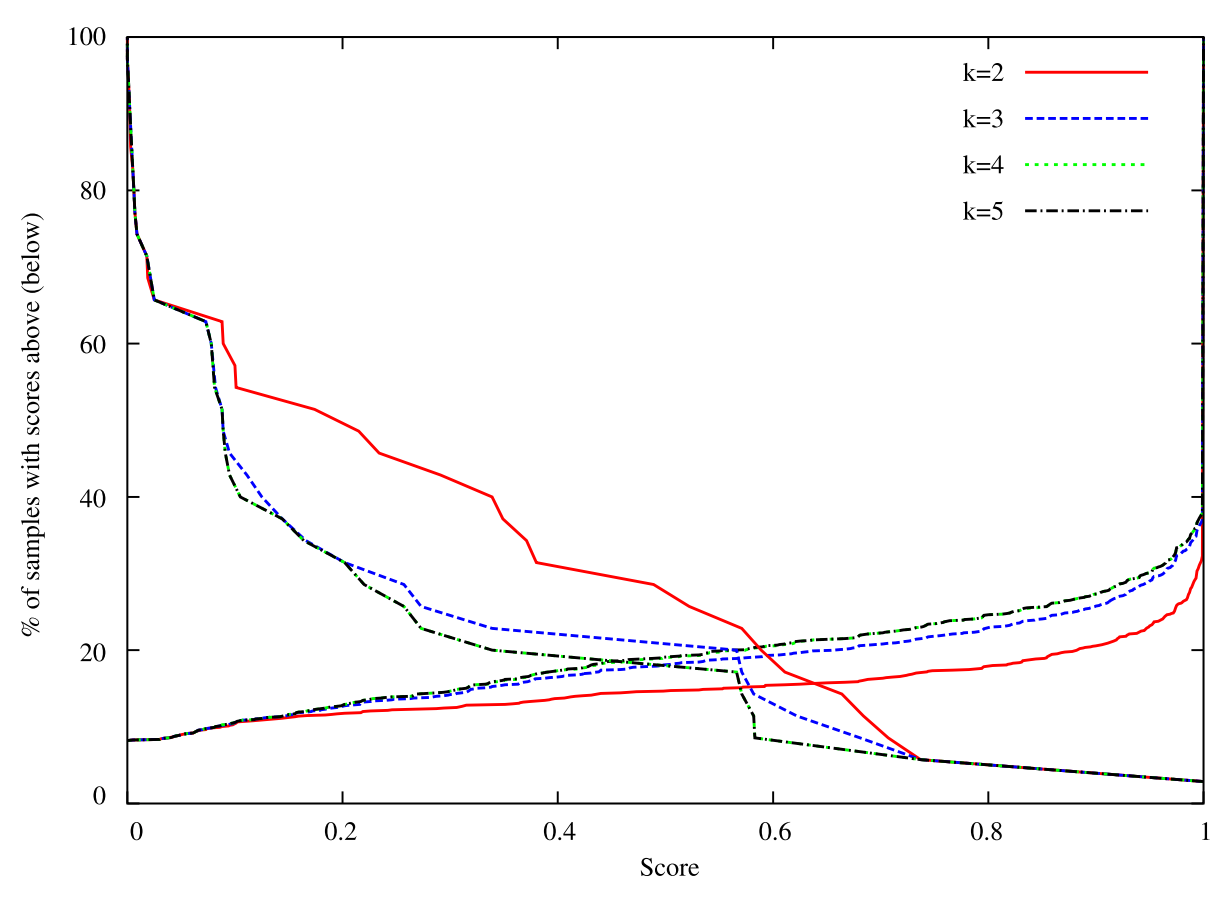

Fig. 6 Malware and goodware recognition. Timeouts for generating the dependency graphs were $120 \mathrm{sec}$ for malware test and training sets and $800 \mathrm{sec}$ for the goodware test set in the figure on the left. The training set consists of $50 \%$ of the entire malware set, chosen at random. The test set consists of the remaining malware samples (curves rising from left to right), and the goodware set (curves falling from left to right). The rising curves represent the percentage of malware samples for which the computed score was less than the corresponding value on the $x$ axis. The falling curves represent the percentage of goodware samples for which the score was greater than the corresponding value on the $x$ axis. The figure shows curves for four different values of $k$, there is essentially no difference between the cases when $k=4$ and $k=5$. For the rising curves, the lowest curve is for $k=2$, the next higher one for $k=3$, and the two highest ones for the remaining cases. For the falling curves, the ordering is reversed. The optimal score for distinguishing malware from goodware is the lowest intersection of the rising and falling curves for the same $k$

diversity of graphs within a single family will produce a classifier that accepts many different behaviors. In Fig. 7, the vertical line of dots above family 25 shows that the classifier learned from half of the samples of family 25 is indeed fairly general and produces large scores for many other families. We could improve both the classification and detection results by eliminating common graphs from the training sets, as done by Christodorescu et al. [9]. Such elimination can be done efficiently in polynomial time.

The pronounced diagonal in Fig. 7 shows that our inferred automata clearly have a significant classification power and could be used to classify malware into families. There is some noise as well. The noise could be attributed to many factors: over-generalization, over- and under-tainting of our DTA [6, 20], insufficiently large dependency graphs, frequently used dynamic libraries that are shared by many applications and malware, and a somewhat ad-hoc pre-classification by the antivirus tools.

\section{Limitations}

There are several inherent limitations of our approach. An attacker could try to mask syscall dependencies so as to be similar (or the same) as those of benign applications. This class of 


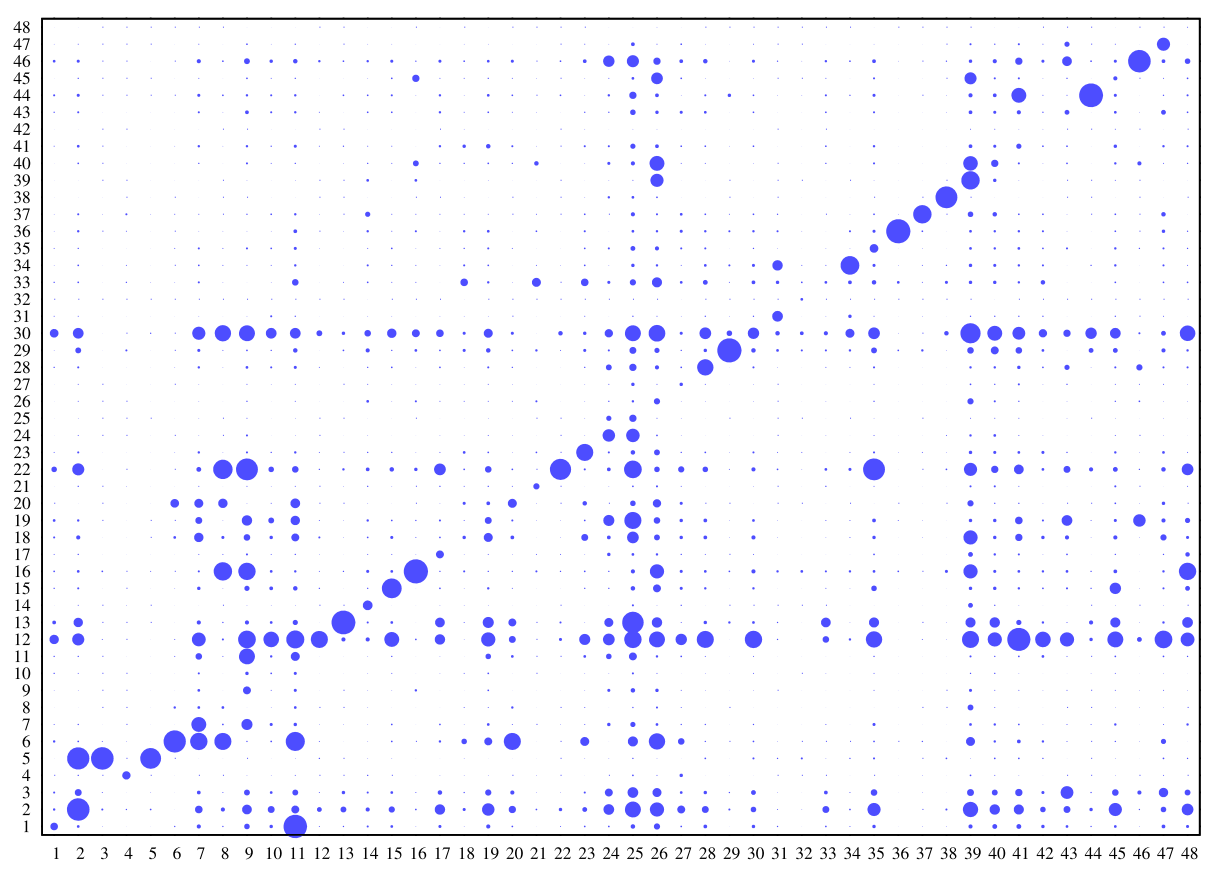

Fig. 7 Malware classification results. The $x(y)$ axis represents the training (test) sets. The size of the shaded circle is proportional to the score computed by Eq. (1). The largest dots correspond to the score of 1

attacks are known as mimicry attacks [35]. All intrusion and behavioral malware detection approaches are susceptible to mimicry attacks. One way to make this harder for the attacker, is to make the analysis more precise, as will be discussed in the following section.

Triggering interesting malware behavior is another challenge. Some behaviors could be triggered only under certain conditions (date, web site visited, choice of the default language, users' actions, ... ). Moser et al. [5, 30] proposed DART [17] as a plausible approach for detecting rarely exhibited behaviors.

As discussed earlier, our DTA environment slows the execution several thousand times, which is obviously too expensive for real-time detection. A lot of work on malware analysis is done in the lab setting, where this is not a significant constraint, but efficiency obviously has to be improved if taint-analysis based approaches are ever to be broadly used for malware detection. Hardware taint-analysis accelerators are a viable option [12, 32], but we also expect we could probably achieve an order of magnitude speedup of our DTA environment with a very careful optimization.

\section{Conclusions and future work}

In this paper, we presented a novel approach to detecting likely malicious behaviors and malware classification based on tree automata inference. We showed that inference, unlike simple matching of dependency graphs, does generalize from the learned patterns and therefore improves detection of yet unseen polymorphic malware samples. We proposed an improved $k$-testable tree automata inference algorithm and showed how the $k$ factor can 
be used as a knob to tune the abstraction level. In our experiments, our approach detects $80 \%$ of the previously unseen polymorphic malware samples, with a $5 \%$ false positive rate, measured on a diverse set of benign applications.

Currently, detection and classification of malware require significant amounts of manual work (see [22] for discussion and references). The goal of our approach is to automate these processes in the laboratory setting. Currently, tracing targeted applications and tracking their syscall dependencies incurs a significant slowdown, which is, in our view, the most significant obstacle to adopting our approach in a real-time real-world setting. We expect that further research and recent progress in hardware-assisted [12, 32] taint analysis could bridge the performance gap.

There are many directions for further improvements. The classification power of our approach could be improved by a more precise analysis of syscall parameters (e.g., using their actual values in the analysis), by dynamically detecting the best value of the $k$ factor in order to match the size of loop bodies that produce patterns in the dependency graphs, by using goodware dependency graphs as negative examples during training, and by combining our approach with the leap mining approach [14].

Also, in the current dependency graphs analysis, we do not distinguish how syscalls return values. For example, if a syscall returns two values, one through the first out parameter and another one through the second, we consider these two values to be the same during the inference, even though our taint analysis distinguishes them. In other words, the inference merges all outputs into a single output and all dependencies are analyzed with respect to that single merged output.

Another interesting direction is inference of more expressive tree languages. Inference of more expressive languages might handle repeated patterns more precisely, generalizing only as much as needed to fold a repeatable pattern into a loop in the tree automaton. Further development of similar methods could have a broad impact in security, forensics, detection of code theft, and perhaps even testing and verification, as the inferred automata can be seen as high-level abstractions of program's behavior.

Acknowledgements We are grateful to Matt Fredrikson and Somesh Jha for sharing their library of classified malware [14] with us. We thank Emiliano Martinez Contreras for giving us an account at virustotal.com that we used to double-check the classification, and for giving us support in using the API through which our experimentation scripts communicated with virustotal.com. We would especially like to thank Lorenzo Martignoni, who wrote the libwst library [28] for extracting and parsing arguments of Windows's system calls. We also thank reviewers for their insightful and constructive comments.

\section{References}

1. Aho AV, Sethi R, Ullman JD (1986) Compilers: principles, techniques, and tools. Addison-Wesley Longman, Boston

2. Babić D (2008) Exploiting structure for scalable software verification. PhD thesis, University of British, Columbia, Vancouver, Canada

3. Babić D, Reynaud D, Song D (2011) Malware analysis with tree automata inference. In: CAV'11: proceedings of the 23rd int conference on computer aided verification. Lecture notes in computer science, vol 6806. Springer, Berlin, pp 116-131

4. Bonfante G, Kaczmarek M, Marion J-Y (2009) Architecture of a morphological malware detector. J Comput Virol 5:263-270

5. Brumley D, Hartwig C, Zhenkai Liang JN, Song D, Yin H (2008) Automatically identifying triggerbased behavior in malware. In: Botnet detection countering the largest security threat. Advances in information security, vol 36. Springer, Berlin, pp 65-88

6. Chow J, Pfaff B, Garfinkel T, Christopher K, Rosenblum M (2004) Understanding data lifetime via whole system simulation. In: Proc of 13th USENIX security symposium 
7. Christodorescu M, Jha S (2004) Testing malware detectors. In: ISSTA'04: proc of the 2004 ACM SIGSOFT int symp on software testing and analysis. ACM Press, New York, pp 34-44

8. Christodorescu M, Jha S, Seshia SA, Song D, Bryant RE (2005) Semantics-aware malware detection. In: SP'05: proc of the 2005 IEEE symp. on security and privacy. IEEE Computer Society Press, Los Alamitos, pp 32-46

9. Christodorescu M, Jha S, Kruegel C (2007) Mining specifications of malicious behavior. In: Proc of the 6th joint meeting of the European software engineering conf and the ACM SIGSOFT symp on the foundations of software engineering. ACM Press, New York, pp 5-14

10. Comon H, Dauchet M, Gilleron R, Löding C, Jacquemard F, Lugiez D, Tison S, Tommasi M (2007) Tree automata techniques and applications. http://tata.gforge.inria.fr/

11. Cormen TH, Leiserson CE, Rivest RL, Stein C (2001) Introduction to algorithms, 2nd edn. The MIT Press, Cambridge

12. Crandall J, Chong F (2005) Minos: control data attack prevention orthogonal to memory model. In: Proc of the 37th int symp on microarchitecture. IEEE Press, New York, pp 221-232

13. Forrest S, Hofmeyr SA, Somayaji A, Longstaff TA (1996) A sense of self for Unix processes. In: Proc of the 1996 IEEE symp on security and privacy. IEEE Computer Society Press, Los Alamitos, pp 120-129

14. Fredrikson M, Jha S, Christodorescu M, Sailer R, Yan X (2010) Synthesizing near-optimal malware specifications from suspicious behaviors. In: Proc of the 2010 IEEE symposium on security and privacy. IEEE Computer Society Press, Los Alamitos, pp 45-60

15. García P (1993) Learning $k$-testable tree sets from positive data. Technical report, Dept. Syst. Inform. Comput., Univ. Politecnica Valencia, Valencia, Spain

16. García P, Vidal E (1990) Inference of k-testable languages in the strict sense and application to syntactic pattern recognition. IEEE Trans Pattern Anal Mach Intell 12:920-925

17. Godefroid P, Klarlund N, Sen K (2005) DART: directed automated random testing. In: PLDI'05: proc of the ACM SIGPLAN conf on prog lang design and implementation. ACM Press, New York, pp 213-223

18. Gold EM (1978) Complexity of automaton identification from given data. Inf Control 37(3):302-320

19. Holzer A, Kinder J, Veith H (2007) Using verification technology to specify and detect malware. In: Lecture notes in computer science, vol 4739. Springer, Berlin, pp 497-504

20. Kang MG, McCamant S, Poosankam P, Song D (2011) DTA++: dynamic taint analysis with targeted control-flow propagation. In: Proceedings of the 18th annual network and distributed system security symposium, San Diego, CA

21. Khoussainov B, Nerode A (2001) Automata theory and its applications. Birkhauser, Basel

22. Kinder J, Katzenbeisser S, Schallhart C, Veith H (2005) Detecting malicious code by model checking. In: Julisch K, Krügel C (eds) GI SIG SIDAR conference on detection of intrusions and malware and vulnerability assessment. Lecture notes in computer science, vol 3548. Springer, Berlin, pp 174-187

23. King JC (1976) Symbolic execution and program testing. Commun ACM 19(7):385-394

24. Knuutila T (1993) Inference of $k$-testable tree languages. In: Bunke H (ed) Advances in structural and syntactic pattern recognition: proc of the int workshop. World Scientific, Singapore, pp 109-120

25. Kolbitsch C, Milani P, Kruegel C, Kirda E, Zhou X, Wang X (2009) Effective and efficient malware detection at the end host. In: The 18th USENIX security symposium

26. López D, Sempere JM, García P (2004) Inference of reversible tree languages. IEEE Trans Syst Man Cybern, Part B, Cybern 34(4):1658-1665

27. Luk C, Cohn R, Muth R, Patil H, Klauser A, Lowney G, Wallace S, Reddi V, Hazelwood K (2005) Pin: building customized program analysis tools with dynamic instrumentation. In: PLDI'05: proc of the 2005 ACM SIGPLAN conf on prog lang design and impl. ACM Press, New York, pp 190-200

28. Martignoni L, Paleari R (2010) The libwst library (a Part of WUSSTrace)

29. Matrosov A, Rodionov E, Harley D, Malcho J (2010) Stuxnet under the microscope. Technical report, Eset

30. Moser A, Kruegel C, Kirda E (2007) Exploring multiple execution paths for malware analysis. In: SP'07: proc of the 2007 IEEE symposium on security and privacy, Washington, DC, USA. IEEE Computer Society Press, Los Alamitos, pp 231-245

31. Newsome J, Song D (2005) Dynamic taint analysis: automatic detection, analysis, and signature generation of exploit attacks on commodity software. In: Proc of the network and distributed systems security symposium

32. Suh G, Lee J, Zhang D, Devadas S (2004) Secure program execution via dynamic information flow tracking. Oper Syst Rev 38(5):85-96

33. Symantec (2010) Symantec global internet security threat report: trends for 2009, vol xv. Technical report, Symantec, April 
34. Wagner D, Dean D (2001) Intrusion detection via static analysis. In: Proc of the 2001 IEEE symposium on security and privacy. IEEE Computer Society Press, Los Alamitos, p 156

35. Wagner $\mathrm{D}$, Soto $\mathrm{P}$ (2002) Mimicry attacks on host-based intrusion detection systems. In: CCS' 02 : proc of the 9th ACM conf on comp and comm security. ACM Press, New York, pp 255-264

36. You I, Yim K (2010) Malware obfuscation techniques: a brief survey. In: Int conf on broadband, wireless computing, communication and applications, pp 297-300

37. Zalcstein Y (1972) Locally testable languages. J Comput Syst Sci 6(2):151-167 